\title{
Approximation Results for Parameter Estimation in Nonlinear Elastomers
}

\author{
H.T. Banks \\ Center for Research in Scientific Computation \\ North Carolina State University \\ Raleigh, NC 27695-8205 \\ Gabriella A. Pinter \\ Department of Mathematics \\ Texas Tech University \\ Lubbock, TX 79409
}

\begin{abstract}
In this paper we present an approximation framework and theoretical convergence results for a class of parameter estimation problems for general abstract nonlinear hyperbolic systems. These systems include as a special case those modeling a large class of nonlinear elastomers.
\end{abstract}

\section{Introduction}

We consider the following class of abstract nonlinear damped parameter dependent hyperbolic systems evolving in a complex separable Hilbert space $H$ :

$$
\begin{aligned}
& w_{t t}+\mathcal{A}_{1}(q) w+\mathcal{A}_{2}(q) w_{t}+\mathcal{N}^{*} g(q)(\mathcal{N} w)=f(t ; q) \\
& w(0)=\varphi_{0} \\
& w_{t}(0)=\varphi_{1} .
\end{aligned}
$$

Here $\mathcal{A}_{1}(q), \mathcal{A}_{2}(q)$ are unbounded operators depending on some parameter $q, g(q)$ is a parameter dependent nonlinear operator in $H, \mathcal{N}$ is an unbounded operator, and $f$ is a parameter dependent forcing term. Precise conditions on these operators are given below. 
This class of systems was introduced in [BGS, BLMY] and further studied in [BLGMY] as a model for the behavior of nonlinear elastomers. These materials, which are used in the development of active and passive vibration devices, are rubber or polymer based composites that involve complex viscoelastic materials. Their behavior cannot be adequately modelled using the theory of linear elasticity. Indeed, they exhibit nonlinearities in material and geometric properties so that there is a nonlinear relationship between stress and strain even for small strains. We illustrate with a simple example that takes into account these nonlinearities, and describe the associated general parameter estimation problems. (For detailed discussions of this and other models see [BLMY, BGS, BLGMY, BL].)

Consider an isotropic, incompressible rubber-like rod under simple elongation with a finite applied stress in the principal axis direction $x_{1}=x$. Let $w$ denote deformation in the $x$ direction. Following the derivation of the model in [BLMY, BGS, BL] we arrive at the partial differential equation

$$
\rho A \frac{\partial^{2} w}{\partial t^{2}}-\frac{\partial}{\partial x}\left(\frac{E A}{3} \frac{\partial w}{\partial x}\right)-\frac{\partial}{\partial x}\left(\frac{E A}{3} \tilde{g}\left(\frac{\partial w}{\partial x}\right)\right)=F,
$$

where $\rho$ is mass density, $\mathrm{E}$ is the generalized modulus of elasticity, $\mathrm{A}$ is the cross sectional area, and $\mathrm{F}$ is an applied external force. If one assumes that the rod is composed of a neoHookean material (see [BL]), then the nonlinearity $\tilde{g}$ in (1.4) is given by $\tilde{g}(\xi)=1-\frac{1}{(1+\xi)^{2}}$ for $-1<\xi<1$. Assuming that we have a slender rod of length $\ell$ that satisfies $w(t, 0)=$ $w(t, \ell)=0$, and defining $V=H_{0}^{1}(0, \ell)$ and $H=L^{2}(0, \ell)$, we obtain the usual Gelfand triple $V \hookrightarrow H \approx H^{*} \hookrightarrow V^{*}$ where $V^{*}=H^{-1}(0, \ell)$. Then equation (1.4) with the specified boundary conditions can be written in the variational form:

$$
\rho A w_{t t}+\mathcal{A}_{1} w+D^{*} \tilde{g}(D w)=F \quad \text { in } V^{*},
$$

where $\mathcal{A}_{1} \in \mathcal{L}\left(V, V^{*}\right)$ is given by

$$
\left\langle\mathcal{A}_{1} \varphi, \psi\right\rangle_{V^{*}, V}=\left\langle\frac{E A}{3} D \varphi, D \psi\right\rangle_{H}
$$

and $D=\frac{\partial}{\partial x} \in \mathcal{L}(V, H)$ is the spatial differentiation operator. For a realistic model we also must include some type of material damping which is known to be present in elastomers (indeed in all materials). Here we assume an internal damping of the form $\mathcal{A}_{2} w_{t}$, where $\mathcal{A}_{2} \in \mathcal{L}\left(V_{2}, V_{2}^{*}\right)$ and $V \hookrightarrow V_{2} \hookrightarrow H$. In the case of Kelvin-Voigt damping we define $V_{2}=V=$ $H_{0}^{1}(0, \ell)$ and

$$
\left\langle\mathcal{A}_{2} \varphi, \psi\right\rangle_{V_{2}^{*}, V_{2}}=\left\langle c_{D} D \varphi, D \psi\right\rangle_{H}
$$


where $c_{D} \in L_{\infty}(0, \ell)$. (We remark that the exact form of the damping mechanism in elastomers is not known and, indeed, is the subject of current research.) With the damping included, we find that our model in variational form for the neo-Hookean elastomer rod is given by

$$
\rho A w_{t t}+\mathcal{A}_{1} w+\mathcal{A}_{2} w_{t}+D^{*} \hat{g}(D w)=F \quad \text { in } V^{*} .
$$

If this model is to be used for simulation or control of the behavior of the elastomer rod we need values for $\rho, E, A, c_{D}, F, \ell$. Some of these can be given or measured explicitly (e.g., $A, \ell, F$ ), or can be found from manufacturers specifications (so-called "book-values"). However, some parameters (e.g., E, $c_{D}$ ) cannot be measured or obtained this way. Also, the "book-values" can vary considerably between samples. Thus we need a method to estimate these "unknown" parameters by dynamic experiments with the sample itself. Moreover, the nonlinearity $\tilde{g}$ is in general unknown and must be estimated (the neo-Hookean assumption is only a first approximation to actual material properties) or chosen from a general class of admissible nonlinearities.

In one general parameter estimation formulation equation (1.6) takes the form (1.1)(1.3) where the structural operators $\mathcal{A}_{1}, \mathcal{A}_{2}$, the nonlinearity $g$ and the input $f$ have all been parameterized by a vector (possibly infinite dimensional) parameter $q$ that must be estimated. Here the parameter $q$ takes values from an admissible parameter set $Q$. Suppose that we have a set of measured observations $z=\left\{z_{i}\right\}_{i=1}^{K}$ corresponding to measurements (e.g., displacements, velocities) taken at time $t_{i}$. In a general least squares parameter estimation problem, we seek to minimize the least squares output functional

$$
J(q, z)=\left|\tilde{C}_{2}\left\{\tilde{C}_{1}\left\{w\left(t_{i}, \cdot ; q\right)\right\}-\left\{z_{i}\right\}\right\}\right|^{2}
$$

over $q \in Q$, where $\left\{w\left(t_{i}, \cdot ; q\right)\right\}$ are the parameter dependent solutions of (1.1)-(1.3) evaluated at time $t_{i}, i=1,2, \ldots K$, and $|\cdot|$ is an appropriately chosen Euclidean norm. The operators $\tilde{C}_{1}, \tilde{C}_{2}$ depend on the type of the collected data. For example, if $z_{i}$ is time domain displacement, velocity or acceleration at a point $x$, then $\tilde{C}_{1}$ involves differentiation $(0,1$ or 2 times, respectively) with respect to time followed by pointwise evaluation in $t$ and $x$. The operator $\tilde{C}_{2}$ is the identity in the case of time domain identification, while it is related to the Fourier transform if we consider fitting the data in the frequency domain (see Chapter 5 of [BSW] for details).

In this formulation the minimization problem involves an infinite dimensional state space and (in general) an infinite dimensional admissible parameter set $Q$. To overcome this difficulty and to obtain a computationally tractable method, we use the general ideas described 
in [BSW]. Namely, let $H^{N}$ be finite dimensional subspaces of $H$, and $Q^{M}$ be a sequence of finite dimensional sets approximating the parameter set $Q$. Denote the orthogonal projections of $H$ onto $H^{N}$ by $P^{N}$. One can formulate a family of approximating estimation problems with finite dimensional state spaces and finite dimensional parameter sets in the following way: find $q \in Q^{M}$ which minimizes

$$
J^{N}(q, z)=\left|\tilde{C}_{2}\left\{\tilde{C}_{1}\left\{w^{N}\left(t_{i}, \cdot ; q\right)\right\}-\left\{z_{i}\right\}\right\}\right|^{2},
$$

where $w^{N}(t ; q) \in H^{N}$ is the solution to the finite dimensional approximation of (1.1)-(1.3) given by:

$$
\begin{aligned}
& \left\langle w_{t t}^{N}, \phi\right\rangle_{V_{2}^{*}, V_{2}}+\left\langle\mathcal{A}_{1}(q) w^{N}, \phi\right\rangle_{V^{*}, V}+\left\langle\mathcal{A}_{2} w_{t}^{N}, \phi\right\rangle_{V_{2}^{*}, V_{2}}+\left\langle g(q)\left(\mathcal{N} w^{N}\right), \mathcal{N} \phi\right\rangle= \\
& \langle f(t ; q), \phi\rangle_{V_{2}^{*}, V_{2}} \\
& w^{N}(0)=P^{N} \varphi_{0}, w_{t}^{N}(0)=P^{N} \varphi_{1}
\end{aligned}
$$

for all $\varphi \in H^{N}$.

Solution of these approximate estimation problems (1.7)-(1.9) provides one with a sequence of parameter estimates $\left\{\bar{q}^{N, M}\right\}$. The crucial question is when one can guarantee that this sequence (or some subsequence) converges to a solution of the original infinite dimensional parameter estimation problem. Under certain suitable assumptions on the approximating spaces $H^{N}$ and approximating sets $Q^{M}$ this question is answered in [BSW] for linear systems and here we extend these ideas to include nonlinear systems.

To permit use of the method outlined above we must be certain that the above systems (1.1)-(1.3) and (1.8)-(1.9) have solutions in some sense for each $q \in Q$. This well-posedness problem (without considering the parameter dependent case) was solved in the recent paper [BGS]. In the following section we summarize these results and give precise conditions under which (1.1)-(1.3) has a unique weak solution for each $q \in Q$. Then in Section 3 we give assumptions on the general parameter estimation problem that we shall use to prove convergence. We also recall Theorem 5.1 from [BSW] that provides a sufficient condition for the convergence of the solutions $\left\{\bar{q}^{N, M}\right\}$ of the approximate estimation problems to a solution of the original parameter estimation problem. Then in Section 4 we show that this condition is satisfied in our case under natural assumptions on the parameter dependence of $\mathcal{A}_{1}, \mathcal{A}_{2}, g$ and $f$. 


\section{Formulation of the Problem}

We assume that there is a sequence of separable Hilbert spaces $V, V_{2}, H, V^{*}, V_{2}^{*}$ forming a Gelfand quintuple [BIW, Wl] satisfying

$$
V \hookrightarrow V_{2} \hookrightarrow H \hookrightarrow V_{2}^{*} \hookrightarrow V^{*}
$$

where we assume that the embedding $V \hookrightarrow V_{2}$ is dense and continuous with $|\varphi|_{V_{2}} \leq c|\varphi|_{V}$ for $\varphi \in V$ and $V_{2} \hookrightarrow H$ is a dense compact embedding with $|\varphi| \leq \tilde{c}|\varphi|_{V_{2}}$. The norm in $H$ will be denoted by $|\cdot|$ while those in $V, V_{2}$ etc. will carry an appropriate subscript. We denote by $\langle,\rangle_{V *, V}$, etc., the usual duality products [W1]. These duality products are the extensions by continuity of the inner product in $H$, denoted by $\langle$,$\rangle throughout. Let$ $Q$ be an infinite dimensional parameter set. The operators $\mathcal{A}_{1}(q)$ and $\mathcal{A}_{2}(q)$ are defined in terms of their sesquilinear forms $\sigma_{1}(q): V \times V \rightarrow \mathbb{C}$ and $\sigma_{2}(q): V_{2} \times V_{2} \rightarrow \mathbb{C}$. That is, $\mathcal{A}_{1}(q) \in \mathcal{L}\left(V, V^{*}\right), \mathcal{A}_{2}(q) \in \mathcal{L}\left(V_{2}, V_{2}^{*}\right)$ and $\left\langle\mathcal{A}_{1}(q) \varphi, \psi\right\rangle_{V^{*}, V}=\sigma_{1}(q)(\varphi, \psi),\left\langle\mathcal{A}_{2}(q) \varphi, \psi\right\rangle_{V_{2}^{*}, V_{2}}=$ $\sigma_{2}(q)(\varphi, \psi)$.

Let $\mathcal{L}_{T}$ denote the space of functions $w:[0, T] \rightarrow H$ such that

$$
w \in C_{W}\left([0, T], V_{2}\right) \cap L^{\infty}([0, T], V)
$$

(the subscript $W$ denotes weak continuity), and

$$
w_{t} \in C_{W}([0, T], H) \cap L^{2}\left([0, T], V_{2}\right),
$$

where the time derivative $w_{t}$ is understood in the sense of distributions with values in a Hilbert Space (see, e.g., [Li1]). The space $\mathcal{L}_{T}$ is equipped with the norm

$$
|w|_{\mathcal{L}_{T}}=\operatorname{ess} \sup _{t \in[0, T]}\left(\left|w_{t}(t)\right|+|w(t)|_{V}\right)+\left(\int_{0}^{T}\left|w_{t}(t)\right|_{V_{2}}^{2} d t\right)^{1 / 2}
$$

Definition 2.1 We say that $w \in \mathcal{L}_{T}$ is a weak solution of the problem (1.1) - (1.3) if it satisfies the equation:

$$
\begin{aligned}
& \int_{0}^{t}\left[-\left\langle w_{\tau}(\tau), \eta_{\tau}(\tau)\right\rangle+\sigma_{1}(q)(w(\tau), \eta(\tau))+\sigma_{2}(q)\left(w_{\tau}(\tau), \eta(\tau)\right)+\right. \\
& +\langle g(q)(\mathcal{N} w(\tau)), \mathcal{N} \eta(\tau)\rangle] d \tau+\left\langle w_{t}(t), \eta(t)\right\rangle= \\
& =\left\langle\varphi_{1}, \eta(0)\right\rangle+\int_{0}^{t}\langle f(\tau ; q), \eta(\tau)\rangle_{V_{2}^{*}, V_{2}} d \tau
\end{aligned}
$$


for any $t \in[0, T]$ and any $\eta \in \mathcal{L}_{T}$, as well as the initial condition

$$
w(0)=\varphi_{0}
$$

Equivalently, $w$ is a weak solution if

$$
\left\langle w_{t t}, \eta\right\rangle_{V^{*}, V}+\sigma_{1}(q)(w, \eta)+\sigma_{2}(q)\left(w_{t}, \eta\right)+\langle g(q)(\mathcal{N} w), \mathcal{N} \eta\rangle=\langle f(q), \eta\rangle_{V_{2}^{*}, V_{2}}
$$

is satisfied for all $\eta \in \mathcal{L}_{T}$ and for almost all $t \in[0, T]$.

To establish our parameter estimation convergence results, we first make the following assumptions (these assumptions are the same as in [BGS] except that here we require them to be satisfied uniformly for all $q \in Q$ ) which will guarantee well-posedness for all $q \in Q$.

A1) The form $\sigma_{1}(q)$ is a Hermitian sesquilinear form: for $\varphi, \psi \in V$

$$
\sigma_{1}(q)(\varphi, \psi)=\overline{\sigma_{1}(q)(\psi, \varphi)} \text { for every } q \in Q \text {. }
$$

A2) The form $\sigma_{1}(q)$ is $V$ bounded: for $\varphi, \psi \in V$

$$
\left.\left|\sigma_{1}(q)(\varphi, \psi) \leq c_{1}\right| \varphi\right|_{V}|\psi|_{V} \text { for every } q \in Q \text {. }
$$

A3) The form $\sigma_{1}(q)$ is strictly $V$ coercive: for $\varphi \in V$

$$
\operatorname{Re} \sigma_{1}(q)(\varphi, \varphi)=\sigma_{1}(q)(\varphi, \varphi) \geq k_{1}|\varphi|_{V}^{2}, \quad k_{1}>0
$$

for every $q \in Q$.

A4) The form $\sigma_{2}(q)$ is $V_{2}$ bounded: for $\varphi, \psi \in V_{2}$

$$
\left|\sigma_{2}(q)(\varphi, \psi)\right| \leq c_{2}|\varphi|_{V_{2}}|\psi|_{V_{2}} \text { for every } q \in Q
$$

A5) The real part of $\sigma_{2}(q)$ is $V_{2}$ coercive and is symmetric:

$$
\begin{aligned}
& \operatorname{Re} \sigma_{2}(q)(\varphi, \varphi)+\lambda_{0}|\varphi|^{2} \geq k_{2}|\varphi|_{V_{2}}^{2} \quad k_{2}>0, \lambda_{0} \geq 0 \\
& \operatorname{Re} \sigma_{2}(q)(\varphi, \psi)=\operatorname{Re} \sigma_{2}(q)(\psi, \varphi), \text { for any } \varphi, \psi \in V_{2}, q \in Q .
\end{aligned}
$$

A6) The forcing term $f(q)$ satisfies $f \in L^{2}\left([0, T], V_{2}^{*}\right)$ for every $q \in Q$. 
A7) The operator $\mathcal{N}$ satisfies

$$
\mathcal{N} \in \mathcal{L}\left(V_{2}, H\right) \text { with }|\mathcal{N} \varphi| \leq \sqrt{\tilde{k}}|\varphi|_{V_{2}}
$$

and the range of $\mathcal{N}$ on $V$ is dense in $H$.

Note that (2.12) and $V \hookrightarrow V_{2}$ implies

$$
\mathcal{N} \in \mathcal{L}(V, H) \text { with }|\mathcal{N} \varphi| \leq \sqrt{k}|\varphi|_{V}
$$

with $k=c^{2} \tilde{k}$.

A8) The nonlinear function $g(q): H \rightarrow H$ is a continuous nonlinear mapping of real gradient (or potential) type. This means that there exists a continuous Frechet-differentiable nonlinear functional $G(q): H \rightarrow \mathbb{R}^{1}$, whose Frechet derivative $G^{\prime}(q)(\varphi) \in \mathcal{L}\left(H, \mathbb{R}^{1}\right)$ at any $\varphi \in H$ can be represented in the form

$$
G^{\prime}(q)(\varphi) \psi=\operatorname{Re}\langle g(q)(\varphi), \psi\rangle \quad \text { for any } \psi \in H
$$

We also require that there are constants $C_{1}, C_{2}, C_{3}$ and $\varepsilon>0$ such that

$$
-\frac{1}{2} k^{-1}\left(k_{1}-\varepsilon\right)|\varphi|^{2}-C_{1} \leq G(q)(\varphi) \leq C_{2}|\varphi|^{2}+C_{3}
$$

for every $q \in Q$, where $k$ is from (2.13) and $k_{1}$ from (2.8).

A9) The nonlinear function $g(q)$ also satisfies

$$
|g(q)(\varphi)| \leq \tilde{C}_{1}|\varphi|+\tilde{C}_{2}, \quad \varphi \in H,
$$

for every $q \in Q$, for some constants $\tilde{C}_{1}, \tilde{C}_{2}$.

An additional condition is necessary for uniqueness of solutions.

A10) For any $\varphi \in H$ the Frechet derivative of $g(q)$ exists and satisfies

$$
g^{\prime}(q)(\varphi) \in \mathcal{L}(H, H) \text { with }\left|g^{\prime}(q)(\varphi)\right|_{\mathcal{L}(H, H)} \leq \widetilde{C}_{3} \text { for every } q \in Q
$$

A11) We assume that for any $u, v \in \mathcal{L}_{T}$, the following inequality is satisfied for any $t \in$ $[0, T], q \in Q:$

$$
\begin{aligned}
& \int_{0}^{t}\{\operatorname{Re}\langle g(q)(\mathcal{N} u(\tau))-g(q)(\mathcal{N} v(\tau)), \mathcal{N} u(\tau)-\mathcal{N} v(\tau)\rangle \\
& \left.+k_{1} k^{-1}|\mathcal{N} u(\tau)-\mathcal{N} v(\tau)|^{2}\right\} d t \\
& +a\left(\left(\int_{0}^{t}|u(\tau)-v(\tau)|^{2} d t\right)^{1 / 2}\right) \geq 0
\end{aligned}
$$

where $a(\xi) \geq 0$ is a continuous function in $\xi \geq 0$ such that 
i) $a(0)=0$.

ii) there exists a first derivative such that $a^{\prime}(0)=0$.

Note that $(2.18)$ is satisfied if, for example,

$$
\operatorname{Re}\langle g(q)(\varphi)-g(q)(\psi), \varphi-\psi\rangle+k_{1} k^{-1}|\varphi-\psi|^{2} \geq 0
$$

for any $\varphi, \psi \in H$, where $k$ and $k_{1}$ are the constants in (2.8) and (2.13). Thus if $H=L^{2}(\Omega), \Omega \subset \mathbb{R}^{m}$, so that $g(q): \mathbb{R} \rightarrow \mathbb{R}$, then a sufficient condition for (2.19) is that $g^{\prime}(q)(\xi) \geq-l_{1}$ for some $l_{1}>0$.

In $[\mathrm{BGS}]$ it is shown that

Theorem 2.1 Under conditions A1)-A11) the system (1.1)-(1.3) has a unique weak solution $w \in \mathcal{L}_{T}$ for every initial condition $\left(\varphi_{0}, \varphi_{1}\right) \in V \times H$. The weak solution satisfies

$$
\left\langle w_{t t}, \eta\right\rangle_{V^{*}, V}+\sigma_{1}(q)(w, \eta)+\sigma_{2}(q)\left(w_{t}, \eta\right)+\langle g(q)(\mathcal{N} w), \mathcal{N} \eta\rangle=\langle f(q), \eta\rangle_{V_{2}^{*}, V_{2}}
$$

for all $\eta \in \mathcal{L}_{T}, q \in Q$ and for almost all $t \in[0, T]$. Also, $w \in C_{W}\left([0, T], V_{2}\right), w_{t} \in$ $C_{W}([0, T], H)$ and the weak solution depends continuously on initial conditions.

\section{The General Parameter Estimation Problem}

Assume that we have a set of observations $z=\left\{z_{i}\right\}_{i=1}^{K}$ corresponding to measurements taken at time $t_{i}$. As stated in the Introduction we would like to find a solution for the least squares minimization problem, i.e., find $q \in Q$ that minimizes

$$
J(q, z)=\left|\tilde{C}_{2}\left\{\tilde{C}_{1}\left\{w\left(t_{i}, \cdot ; q\right)\right\}-\left\{z_{i}\right\}\right\}\right|^{2}
$$

where $\left\{w\left(t_{i}, \cdot ; q\right)\right\}$ are the parameter dependent solutions of (1.1)-(1.3) evaluated at time $t_{i}, i=1,2, \ldots K$. To this end, we consider Galerkin type approximations to (1.1)-(1.3) and define a family of approximating parameter estimation problems.

Let $H^{N}$ be finite dimensional subspaces of $H$ and let $Q^{M}$ be finite dimensional sets approximating (in a sense to be made precise below) the parameter set $Q$. Let $P^{N}$ denote the orthogonal projections of $H$ onto $H^{N}$. Then the approximate parameter estimation problems can be stated in the following way: find $q \in Q^{M}$ that minimizes

$$
J^{N}(q, z)=\left|\tilde{C}_{2}\left\{\tilde{C}_{1}\left\{w^{N}\left(t_{i}, \cdot ; q\right)\right\}-\left\{z_{i}\right\}\right\}\right|^{2}
$$


where $w^{N}(t ; q) \in H^{N}$ is the solution to the finite dimensional approximation of (1.1)-(1.3) given by (1.8)-(1.9).

We make the following assumptions for the spaces $H^{N}$ and $H$ and the sets $Q^{M}$ and $Q$ (see $[\mathrm{BSW}]$ ).

B1) The sets $Q$ and $Q^{M}$ lie in a metric space $\tilde{Q}$ with metric $d$. We assume that $Q$ and $Q^{M}$ are compact in this metric and there is a mapping $i^{M}: Q \rightarrow Q^{M}$ such that $Q^{M}=i^{M}(Q)$. Also, for each $q \in Q, i^{M}(q) \rightarrow q$ in $\tilde{Q}$ with the convergence uniform in $q \in Q$.

B2) The finite dimensional subspaces $H^{N}$ satisfy $H^{N} \subset V$.

B3) For each $\psi \in V,\left|\psi-P^{N} \psi\right|_{V} \rightarrow 0$ as $N \rightarrow \infty$.

B4) For each $\psi \in V_{2},\left|\psi-P^{N} \psi\right|_{V_{2}} \rightarrow 0$ as $N \rightarrow \infty$.

We also assume that $\mathcal{A}_{1}, \mathcal{A}_{2}, g, f$ depend continuously on the parameter $q \in Q$, i.e., they satisfy the following conditions:

C1) $\left|\sigma_{1}(q)(\phi, \psi)-\sigma_{1}(\tilde{q})(\phi, \psi)\right| \leq \gamma_{1} d(q, \tilde{q})|\phi|_{V}|\psi|_{V}$, for every $\phi, \psi \in V$.

C2) $\left|\sigma_{2}(q)(\xi, \eta)-\sigma_{2}(\tilde{q})(\xi, \eta)\right| \leq \gamma_{2} d(q, \tilde{q})|\xi|_{V_{2}}|\eta|_{V_{2}}$, for every $\xi, \eta \in V_{2}$.

C3) $|g(q)(\phi)-g(\tilde{q})(\phi)| \leq \gamma_{3} d(q, \tilde{q})|\phi|$ for all $\phi \in H$.

C4) The mapping $q \rightarrow f(\cdot ; q)$ is continuous from $Q$ to $L^{2}\left([0, T], V_{2}^{*}\right)$.

Under conditions A1)-A11), B1), C1)-C4) we know that a solution $\left\{\bar{q}^{N, M}\right\}$ to the approximate parameter estimation problem (1.7)-(1.9) and a solution $\bar{q}$ to the original parameter estimation problem for (3.1) exist. A general sufficient condition for the convergence of $\left\{\bar{q}^{N, M}\right\}$ to $\bar{q}$ is given in Theorem 5.1 of $[\mathrm{BSW}]$ (see also $[\mathrm{BK}]$ ):

Theorem 3.1 To obtain convergence of at least a subsequence of $\left\{\bar{q}^{N, M}\right\}$ to a solution $\bar{q}$ of minimizing (3.1) subject to (1.1)-(1.3), it suffices, under assumption B1), to argue that for arbitrary sequences $\left\{q^{N, M}\right\}$ in $Q^{M}$ with $q^{N, M} \rightarrow q \in Q$, we have

$$
\tilde{C}_{2} \tilde{C}_{1} w^{N}\left(t ; q^{N, M}\right) \rightarrow \tilde{C}_{2} \tilde{C}_{1} w(t ; q) .
$$




\section{Convergence Results}

In this section we show that under our general conditions given above, the convergence criteria (3.3) of Theorem 3.1 holds for a reasonable class of observation operators $\tilde{C}_{1}, \tilde{C}_{2}$.

Theorem 4.1 Suppose that assumptions A1)-A11), B1)-B4) and (1)-C4) are satisfied. Let $q^{N}$ be arbitrary in $Q^{N}$ such that $q^{N} \rightarrow q \in Q$ as $N \rightarrow \infty$. Then we have

$$
w^{N}\left(t, q^{N}\right) \rightarrow w(t, q) \text { in } V, \text { for all } t \geq 0
$$

and

$$
w_{t}^{N}\left(t, q^{N}\right) \rightarrow w_{t}(t, q) \text { in } H \text { for all } t \geq 0 \text { and in } V_{2} \text { for almost all } t \geq 0,
$$

where $w^{N}, w_{t}^{N}$ satisfy

$$
\begin{aligned}
& \left\langle w_{t t}^{N}(t), \phi\right\rangle_{V^{*}, V}+\sigma_{2}\left(q^{N}\right)\left(w_{t}^{N}(t), \phi\right)+\sigma_{1}\left(q^{N}\right)\left(w^{N}(t), \phi\right)+\left\langle g\left(q^{N}\right)\left(\mathcal{N} w^{N}\right), \mathcal{N} \phi\right\rangle= \\
& \left\langle f\left(t, q^{N}\right), \phi\right\rangle_{V_{2}^{*}, V_{2}} \\
& w^{N}(0)=P^{N} \varphi_{0}, w_{t}^{N}(0)=P^{N} \varphi_{1}
\end{aligned}
$$

for all $\phi \in H^{N}$, and $w, w_{t}$ satisfy

$$
\begin{aligned}
& \left\langle w_{t t}(t), \phi\right\rangle_{V^{*}, V}+\sigma_{2}(q)\left(w_{t}(t), \phi\right)+\sigma_{1}(q)(w(t), \phi)+\langle g(q)(\mathcal{N} w), \mathcal{N} \phi\rangle= \\
& \langle f(t, q), \phi\rangle_{V_{2}^{*}, V_{2}} \\
& w(0)=\varphi_{0}, w_{t}(0)=\varphi_{1}
\end{aligned}
$$

for all $\phi \in V$.

Proof: We know that $w(t) \in V, w_{t}(t) \in H$ for all $t \geq 0$ and $w_{t}(t) \in V_{2}$ for almost all $t \geq 0$. By the triangle inequality

$$
\left|w^{N}\left(t, q^{N}\right)-w(t, q)\right|_{V} \leq\left|w^{N}\left(t, q^{N}\right)-P^{N} w(t, q)\right|_{V}+\left|P^{N} w(t, q)-w(t, q)\right|_{V}
$$

By assumption B3) the second term on the right side goes to 0 as $N \rightarrow \infty$. So to prove our statement about $w^{N}\left(t, q^{N}\right)$ it is enough to show that the first term on the right side also goes to 0 as $N \rightarrow \infty$. Similarly,

$$
\left|w_{t}^{N}\left(t, q^{N}\right)-w_{t}(t, q)\right|_{V_{2}} \leq\left|w_{t}^{N}\left(t, q^{N}\right)-P^{N} w_{t}(t, q)\right|_{V_{2}}+\left|P^{N} w_{t}(t, q)-w_{t}(t, q)\right|_{V_{2}} .
$$


The last term again goes to 0 by B4), so to prove our statement it is enough to show that the first term also converges to zero.

Let us introduce the following notation:

$$
w^{N}=w^{N}\left(t, q^{N}\right), w=w(t, q)
$$

and

$$
\Delta^{N}=w^{N}\left(t, q^{N}\right)-P^{N} w(t, q)
$$

Then

$$
\begin{gathered}
\Delta_{t}^{N}=w_{t}^{N}-\frac{d}{d t} P^{N} w=w_{t}^{N}-P^{N} w_{t}, \\
\Delta_{t t}^{N}=w_{t t}^{N}-\frac{d^{2}}{d t^{2}} P^{N} w
\end{gathered}
$$

since $w_{t} \in L^{2}\left([0, t], V_{2}\right)$.

From (4.1) and (4.2) we have that for every $\psi \in H^{N}$ :

$$
\begin{aligned}
& \left\langle\Delta_{t t}^{N}, \psi\right\rangle_{V^{*}, V}=\left\langle w_{t t}^{N}-w_{t t}+w_{t t}-\frac{d^{2}}{d t^{2}} P^{N} w, \psi\right\rangle_{V^{*}, V} \\
& =\left\langle f\left(q^{N}\right), \psi\right\rangle_{V_{2}^{*}, V_{2}}-\sigma_{2}\left(q^{N}\right)\left(w_{t}^{N}, \psi\right)-\sigma_{1}\left(q^{N}\right)\left(w^{N}, \psi\right)-\left\langle g\left(q^{N}\right) \mathcal{N} w^{N}, \mathcal{N} \psi\right\rangle \\
& -\langle f(q), \psi\rangle_{V_{2}^{*}, V_{2}}+\sigma_{2}(q)\left(w_{t}, \psi\right)+\sigma_{1}(q)(w, \psi)+\langle g(q) \mathcal{N} w, \mathcal{N} \psi\rangle+ \\
& \left\langle w_{t t}-\frac{d^{2}}{d t^{2}} P^{N} w, \psi\right\rangle_{V^{*}, V}
\end{aligned}
$$

By adding and subtracting we obtain for all $\psi \in H^{N}$

$$
\begin{aligned}
& \left\langle\Delta_{t t}^{N}, \psi\right\rangle_{V^{*}, V}+\sigma_{1}\left(q^{N}\right)\left(\Delta^{N}, \psi\right)=\left\langle w_{t t}-\frac{d^{2}}{d t^{2}} P^{N} w, \psi\right\rangle_{V^{*}, V}-\left\langle f(q)-f\left(q^{N}\right), \psi\right\rangle_{V_{2}^{*}, V_{2}}+ \\
& \sigma_{2}\left(q^{N}\right)\left(w_{t}-P^{N} w_{t}, \psi\right)+\sigma_{2}(q)\left(w_{t}, \psi\right)-\sigma_{2}\left(q^{N}\right)\left(w_{t}, \psi\right)+ \\
& \sigma_{1}\left(q^{N}\right)\left(w-P^{N} w, \psi\right)+\sigma_{1}(q)(w, \psi)-\sigma_{1}\left(q^{N}\right)(w, \psi)- \\
& \sigma_{2}\left(q^{N}\right)\left(\Delta_{t}^{N}, \psi\right)+\langle g(q) \mathcal{N} w, \mathcal{N} \psi\rangle-\left\langle g\left(q^{N}\right) \mathcal{N} w^{N}, \mathcal{N} \psi\right\rangle .
\end{aligned}
$$

We may choose $\psi=\Delta_{t}^{N}$ since $\Delta_{t}^{N} \in H^{N}$. Then $\left\langle\Delta_{t t}^{N}, \Delta_{t}^{N}\right\rangle_{V^{*}, V}=\frac{1}{2} \frac{d}{d t}\left|\Delta_{t}^{N}\right|_{V}^{2}$. As in [BSW] we find:

$$
\begin{aligned}
& \frac{d}{d t}\left(\left\langle\left|\Delta_{t}^{N}\right|_{V}^{2}+\sigma_{1}\left(q^{N}\right)\left(\Delta^{N}, \Delta^{N}\right)\right)=2 \operatorname{Re}\left\{\left\langle w_{t t}-\frac{d^{2}}{d t^{2}} P^{N} w, \Delta_{t}^{N}\right\rangle_{V^{*}, V}\right.\right. \\
& -\left\langle f(q)-f\left(q^{N}\right), \Delta_{t}^{N}\right\rangle_{V_{2}^{*}, V_{2}}+
\end{aligned}
$$




$$
\begin{aligned}
& \sigma_{2}\left(q^{N}\right)\left(w_{t}-P^{N} w_{t}, \Delta_{t}^{N}\right)+\sigma_{2}(q)\left(w_{t}, \Delta_{t}^{N}\right)-\sigma_{2}\left(q^{N}\right)\left(w_{t}, \Delta_{t}^{N}\right) \\
& +\sigma_{1}\left(q^{N}\right)\left(w-P^{N} w, \Delta_{t}^{N}\right)+\sigma_{1}(q)\left(w, \Delta_{t}^{N}\right)-\sigma_{1}\left(q^{N}\right)\left(w, \Delta_{t}^{N}\right) \\
& \left.-\sigma_{2}\left(q^{N}\right)\left(\Delta_{t}^{N}, \Delta_{t}^{N}\right)+\left\langle g(q) \mathcal{N} w, \mathcal{N} \Delta_{t}^{N}\right\rangle-\left\langle g\left(q^{N}\right) \mathcal{N} w^{N}, \mathcal{N} \Delta_{t}^{N}\right\rangle\right\} .
\end{aligned}
$$

We denote the left side of $(4.5)$ by $L(t)$ and the right side by $R(t)$. Integrating $L$ from 0 to t, using initial conditions

$$
\Delta^{N}(0)=w^{N}(0)-P^{N} w(0)=w^{N}(0)-P^{N} \varphi_{0}=0
$$

and

$$
\Delta_{t}^{N}(0)=w_{t}^{N}(0)-P^{N} w_{t}(0)=w_{t}^{N}(0)-P^{N} \varphi_{1}=0,
$$

along with A3), we have

$$
\int_{0}^{t} L(s) d s \geq\left|\Delta_{t}^{N}\right|_{V}^{2}+k_{1}\left|\Delta^{N}\right|_{V}^{2}
$$

We next argue that

$$
\int_{0}^{t} R(s) d s \leq \mu_{1} \tilde{\delta}^{N}(t)+\mu_{2} \int_{0}^{t}\left|\Delta_{t}^{N}\right|_{V}^{2}+k_{1}\left|\Delta^{N}\right|_{V}^{2} d s
$$

where $\tilde{\delta}^{N}(t) \rightarrow 0$ as $N \rightarrow \infty$ and $\mu_{1}, \mu_{2}$ are positive constants. Then by Gronwall's inequality we obtain that

$$
k_{1}\left|\Delta^{N}(t)\right|_{V}^{2}+\left|\Delta_{t}^{N}(t)\right|_{V}^{2} \rightarrow 0 \text { as } N \rightarrow \infty \text { for each } t \geq 0
$$

which implies the desired results.

Proceeding as in $[\mathrm{BSW}]$, we have that

$$
\begin{aligned}
& \int_{0}^{t} R(s) d s \leq \nu_{1} \delta^{N}(t)+\nu_{2} \int_{0}^{t}\left|\Delta_{t}^{N}\right|_{V}^{2}+k_{1}\left|\Delta^{N}\right|_{V}^{2} d s+ \\
& 2 \operatorname{Re} \int_{0}^{t}\left(\left\langle g(q) \mathcal{N} w, \mathcal{N} \Delta_{t}^{N}\right\rangle-\left\langle g\left(q^{N}\right) \mathcal{N} w^{N}, \mathcal{N} \Delta_{t}^{N}\right\rangle\right) d s
\end{aligned}
$$

where

$$
\begin{aligned}
& \delta^{N}(t)=\int_{0}^{t} \operatorname{Re}\left\{\left\langle w_{t t}-\frac{d^{2}}{d t^{2}} P^{N} w, \Delta_{t}^{N}\right\rangle_{V^{*}, V}+\left|f(q)-f\left(q^{N}\right)\right|_{V_{2}^{*}}^{2}+c_{2}^{2}\left|w_{t}-P^{N} w_{t}\right|_{V_{2}}^{2}\right. \\
& \left.\gamma_{2}^{2} d^{2}\left(q, q^{N}\right)\left|w_{t}\right|_{V_{2}}^{2}+c_{2}\left|w-P^{N} w\right|_{V}^{2}+\gamma_{1}^{2} d^{2}\left(q, q^{N}\right)|w|_{V}^{2}\right\} d s,
\end{aligned}
$$

and $\delta^{N}(t) \rightarrow 0$ as $N \rightarrow \infty$ by (5.18) of [BSW], B3), B4), properties of $w, w_{t}$ and assumptions of the theorem. Finally, we need to show that the last integral containing the terms involving 
$g$ can also be estimated from above by an expression similar to the right side of (4.7). We may argue

$$
\begin{aligned}
& \left|\int_{0}^{t}\left\langle g(q) \mathcal{N} w, \mathcal{N} \Delta_{t}^{N}\right\rangle-\left\langle g\left(q^{N}\right) \mathcal{N} w^{N}, \mathcal{N} \Delta_{t}^{N}\right\rangle d s\right| \leq \\
& \left|\int_{0}^{t}\left\langle g(q) \mathcal{N} w-g\left(q^{N}\right) \mathcal{N} w, \mathcal{N} \Delta_{t}^{N}\right\rangle+\left\langle g\left(q^{N}\right) \mathcal{N} w-g\left(q^{N}\right) \mathcal{N} w^{N}, \mathcal{N} \Delta_{t}^{N}\right\rangle d s\right| \leq \\
& \frac{1}{2} \int_{0}^{t}\left(\gamma_{3}^{2} d^{2}\left(q, q^{N}\right) k|w|_{V}^{2}+k\left|\Delta_{t}^{N}\right|_{V}^{2}\right) d s+ \\
& \left|\int_{0}^{t}\left\langle g\left(q^{N}\right) \mathcal{N} w-g\left(q^{N}\right) \mathcal{N} P^{N} w, \mathcal{N} \Delta_{t}^{N}\right\rangle d s\right|+ \\
& \left|\int_{0}^{t}\left\langle g\left(q^{N}\right) \mathcal{N} P^{N} w-g\left(q^{N}\right) \mathcal{N} w^{N}, \mathcal{N} \Delta_{t}^{N}\right\rangle d s\right|
\end{aligned}
$$

Now the first integral on the right is dominated by the right side of (4.7) (with suitably chosen constants). To estimate the last two integrals we use the same method as in [BGS]. We have

$$
\begin{aligned}
& \left|\int_{0}^{t}\left\langle g\left(q^{N}\right) \mathcal{N} w-g\left(q^{N}\right) \mathcal{N} P^{N} w, \mathcal{N} \Delta_{t}^{N}\right\rangle d s\right|= \\
& \left|\int_{0}^{t}\left\langle\int_{0}^{1} g^{\prime}\left(\theta \mathcal{N} w(s)+(1-\theta) \mathcal{N} P^{N} w(s)\right)\left[\mathcal{N} w(s)-\mathcal{N} P^{N} w(s)\right] d \theta, \mathcal{N} \Delta_{t}^{N}\right\rangle d s\right| \leq \\
& \int_{0}^{t} \tilde{C}_{3}\left|\mathcal{N} w-\mathcal{N} P^{N} w\right|\left|\mathcal{N} \Delta_{t}^{N}\right| d s \leq \frac{1}{2} \int_{0}^{t}\left(\tilde{C}_{3}^{2}\left|\mathcal{N} w-\mathcal{N} P^{N} w\right|^{2}+\left|\mathcal{N} \Delta_{t}^{N}\right|^{2}\right) d s \leq \\
& \frac{1}{2} k \int_{0}^{t} \tilde{C}_{3}^{2}\left|w-P^{N} w\right|_{V}^{2} d s+\frac{1}{2} k \int_{0}^{t}\left|\Delta_{t}^{N}\right|_{V}^{2} d s .
\end{aligned}
$$

Here the first term in the last expression goes to 0 as $N \rightarrow \infty$ by B3) and properties of $w$, while the second is dominated by second term on the right side of (4.7). Similarly,

$$
\begin{aligned}
& \left|\int_{0}^{t}\left\langle g\left(q^{N}\right) \mathcal{N} P^{N} w-g\left(q^{N}\right) \mathcal{N} w^{N}, \mathcal{N} \Delta_{t}^{N}\right\rangle d s\right| \leq \\
& \frac{1}{2} k \int_{0}^{t} \tilde{C}_{3}^{2}\left|P^{N} w-w^{N}\right|_{V}^{2}+\left|\Delta_{t}^{N}\right|_{V}^{2} d s \leq \\
& \frac{1}{2} k \int_{0}^{t} \tilde{C}_{3}^{2}\left|\Delta^{N}\right|_{V}^{2}+\left|\Delta_{t}^{N}\right|_{V}^{2} d s,
\end{aligned}
$$

which again is dominated by the right side of (4.7). This completes the required arguments.

We note that the above theorem gives a computationally tractable method to solve the parameter estimation problem involving (3.1) in case the data collected consists of displacement or velocity measurements, i.e., $\tilde{C}_{1}$ is either the identity or differentiation with respect 
to time once followed by evaluation in $t$ and $x$. However, the case of accelerometer data is more complicated, since then Theorem 3.1 requires $w_{t t}^{N}\left(t ; q^{N}\right) \rightarrow w_{t t}(t ; q)$ in $V^{*}$ for $t \in[0, T]$. We will now give conditions under which this convergence can be obtained.

Let us suppose that $V_{2}=V$, i.e., we have strong damping, such as Kelvin-Voigt damping in the example given in the Introduction. We can formulate the system (1.1)-(1.3) in variational form (4.2) and rewrite it in first order vector form on $\mathcal{H}=V \times H$ in the coordinates

$$
z=\left(\begin{array}{c}
z_{1} \\
z_{2}
\end{array}\right)=\left(\begin{array}{c}
w \\
w_{t}
\end{array}\right)
$$

We define $\mathcal{V}=V \times V$ and $\sigma(q): \mathcal{V} \times \mathcal{V} \rightarrow \mathbb{C}$ by

$$
\sigma(q)\left(\left(\begin{array}{c}
\xi \\
\eta
\end{array}\right),\left(\begin{array}{c}
\phi \\
\psi
\end{array}\right)\right)=-\langle\eta, \phi\rangle_{V}+\sigma_{1}(q)(\xi, \psi)+\sigma_{2}(q)(\eta, \psi) .
$$

Then (4.2) can be rewritten as

$$
\begin{aligned}
& \left\langle z_{t}, \Phi\right\rangle+\sigma(q)(z, \Phi)=\langle F(q), \Phi\rangle \text { for all } \Phi \in \mathcal{V} \\
& z(0)=\left(\begin{array}{c}
\varphi_{0} \\
\varphi_{1}
\end{array}\right)
\end{aligned}
$$

where

$$
F(q)=\left(\begin{array}{c}
0 \\
f(q)-\mathcal{N}^{*} g(q)\left(\mathcal{N} z_{1}\right)
\end{array}\right) .
$$

We can also write this in the equivalent operator form (not distinguishing between row and column vectors):

$$
\begin{aligned}
& z_{t}=\mathbf{A}(q) z+F(q) \\
& z(0)=\left(\begin{array}{c}
\varphi_{0} \\
\varphi_{1}
\end{array}\right)
\end{aligned}
$$

where $\sigma(q)(\Phi, \Psi)=\langle-\mathbf{A}(q) \Phi, \Psi\rangle_{\mathcal{V} * \mathcal{V}}$ with

$$
\mathbf{A}(q)=\left[\begin{array}{cc}
0 & I \\
-\mathcal{A}_{1}(q) & -\mathcal{A}_{2}(q)
\end{array}\right] .
$$

It is shown in $[\mathrm{BIW}, \mathrm{BSW}]$ that if $V=V_{2}$, then $\mathbf{A}(q)$ generates an analytic semigroup $S(t ; q)$ on $\mathcal{V}^{*}=V \times V^{*}$. Then the weak solution of (1.1)-(1.3) can be represented in the form:

$$
z(t ; q)=\left(\begin{array}{c}
w(t ; q) \\
w_{t}(t ; q)
\end{array}\right)=S(t ; q)\left(\begin{array}{c}
\varphi_{0} \\
\varphi_{1}
\end{array}\right)+\int_{0}^{t} S(t-\tau ; q) F(\tau ; q) d \tau .
$$


Letting $\mathcal{H}^{N}=H^{N} \times H^{N}$, we can restrict $\sigma(q)$ to $\mathcal{H}^{N} \times \mathcal{H}^{N}$, denote this restriction by $\sigma^{N}$ and define $\mathbf{A}^{N}(q): \mathcal{H}^{N} \rightarrow \mathcal{H}^{N}$ by $\left\langle\mathbf{A}^{N}(q) \Phi, \Psi\right\rangle=\sigma^{N}(\Phi, \Psi)$ for all $\Phi, \Psi \in H^{N} \times H^{N}$. Then $\mathbf{A}^{N}$ generates an analytic semigroup $S^{N}(t ; q)$ on $\mathcal{H}^{N}$. Solutions of (1.8)-(1.9) can then be represented as:

$$
z^{N}(t ; q)=\left(\begin{array}{c}
w^{N}(t ; q) \\
w_{t}^{N}(t ; q)
\end{array}\right)=S^{N}(t ; q)\left(\begin{array}{c}
P^{N} \varphi_{0} \\
P^{N} \varphi_{1}
\end{array}\right)+\int_{0}^{t} S^{N}(t-\tau ; q) P^{N} F(\tau ; q) d \tau
$$

where $P^{N} F$ is understood to mean $\left(\begin{array}{c}P^{N} F_{1} \\ P^{N} F_{2}\end{array}\right)$ if $F_{i}$ are the components of $F$ and $P^{N}$ : $V^{*} \rightarrow H^{N}$ is the generalized projection (in the sense of the duality product). We can then use the theory developed in $[\mathrm{BR}]$ to obtain $z_{t}^{N}\left(t ; q^{N}\right) \rightarrow z_{t}(t ; q)$ when $q^{N} \rightarrow q$. Note that this will guarantee that $w_{t t}^{N}\left(t ; q^{N}\right) \rightarrow w_{t t}(t ; q)$ in $V^{*}$ for $t \in[0, T]$ (which is what we desired). According to Theorem 3.1, 3.2 in [BR] and the remarks following them, this convergence is guaranteed if we can argue convergence of (4.18) to (4.17) after differentiation of these terms with respect to time. Since we have analytic semigroups we obtain this property if $F(q) \in \tilde{L}^{\infty}\left([0, T], \mathcal{V}^{*}\right)$ (i.e., pointwise defined and bounded $\mathcal{V}^{*}$ valued functions) and $P^{N} \varphi \rightarrow \varphi$ in $V^{*}$. Thus, we can state the following theorem:

Theorem 4.2 Let $V=V_{2}$ and $f(q) \in \tilde{L}^{\infty}\left([0, T], V^{*}\right)$ in the system (1.1)-(1.3). Let A1)A11), B1)-B4), C1)-C4) hold. Morover, assume that $P^{N} \varphi \rightarrow \varphi$ in $V^{*}$ for $\varphi \in V^{*}$. Then for any $q^{N} \rightarrow q \in Q$ we have $w_{t t}^{N}\left(t ; q^{N}\right) \rightarrow w_{t t}(t ; q)$ in $V^{*}$ for $t \in[0, T]$.

Proof: Using the arguments in $[\mathrm{BR}]$ with $(4.17)$, (4.18) we only need to argue that $f(\cdot ; q)-$ $\mathcal{N}^{*} g(q)\left(\mathcal{N} z_{1}\right) \in \tilde{L}^{\infty}\left([0, T], V^{*}\right)$. But this follows from the fact that $f(q) \in \tilde{L}^{\infty}\left([0, T], V^{*}\right)$, $z=w \in C_{W}\left([0, T], V_{2}\right)$ and $\mathcal{N} \in \mathcal{L}\left(V_{2}, H\right)$.

\section{Acknowledgement:}

This research was supported in part by the Air Force Office of Scientific Research under grant AFOSR F49620-95-1-0236.

\section{References}

[BGS] H.T.Banks, D.S. Gilliam, and V.I. Shubov, Global solvability for damped nonlinear hyperbolic systems, CRSC-TR95-25. August, 1995; Differential and Integral Equations, to appear. 
[BIW] H. T. Banks, K. Ito, and Y. Wang. Well-posedness for damped second order systems with unbounded input operators, CRSC-TR93-10, June 1993; Differential and Integral Equations, 8(1995), pp. 587-606.

[BK] H.T. Banks and K. Kunisch, Estimation Techniques for Distributed Parameter Systems, Birkhäuser, Boston, 1989.

[BL] H.T. Banks and N.J. Lybeck, Modeling methodology for elastomer dynamics, CRSCTR96-29, September, 1996; Systems and Control in the 21st Century, (ed. by C. Byrnes, B. Datta, D. Gilliam and C. Martin), Birkhäuser, Boston, 1996, pp. 37-50.

[BLGMY] H.T. Banks, N.J. Lybeck, M.J. Gaitens, B.C. Munoz, and L.C. Yanyo, Modeling the dynamic mechanical behavior of elastomers, CRSC-TR96-26, September, 1996; Rubber Chem and Tech., submitted.

[BLMY] H.T. Banks, N.J. Lybeck, B.C. Munoz, and L.C. Yanyo, Nonlinear elastomers: Modeling and estimation, CRSC-TR95-19, May, 1995; Proc. 3rd IEEE Mediterranean Symp. on New Directions in Control, Limassol, Cyprus, July, 1995, Vol. 1. pp. $1-7$.

[BR] H.T. Banks and D.A. Rebnord, Analytic semigroups: Applications to inverse problems for flexible structures, in Differential Equations with Applications in Biology, Physics and Engineering, Marcel Dekker Inc., New York, 1991, pp. 21-35.

[BSW] H.T. Banks, R. C. Smith and Y. Wang, Smart Material Structures, Modeling, Estimation and Control, Masson/Wiley, Paris/Chichester, 1996.

[Li1] J.L. Lions, Optimal Control of Systems Governed by Partial Differential Equations, Springer-Verlag, New York, 1971.

[P] A. Pazy, Semigroups of Linear Operators and Applications to Partial Differential Equations, Springer Verlag, New York, 1983.

[Wl] J. Wloka, Partial Differential Equations, Cambridge Univ. Press, 1992. 